\title{
Catalan and Schröder permutations sortable by two restricted stacks
}

\author{
Jean-Luc Baril ${ }^{\mathrm{a}}$, Giulio Cerbai ${ }^{\mathrm{b}, 1}$, Carine Khalil ${ }^{\mathrm{a}}$, Vincent Vajnovszki ${ }^{\mathrm{a}, *}$ \\ a LIB, Université de Bourgogne Franche-Comté, B.P. 47 870, 21078 Dijon Cedex, France \\ b Dipartimento di Matematica e Informatica “U. Dini”, University of Firenze, Firenze, Italy
}

\section{A R T I C L E I N F O}

\section{Article history:}

Received 14 September 2020

Received in revised form 15 February 2021

Accepted 2 May 2021

Available online 19 May 2021

Communicated by Amit Chakrabarti

\section{Keywords:}

Stack sorting/two stacks in series

Pattern avoiding permutations/machines

Catalan and the Schröder numbers

Combinatorial problems

\begin{abstract}
A B S T R A C T
Pattern avoiding machines were introduced recently by Claesson, Cerbai and Ferrari as a particular case of the two-stacks in series sorting device. They consist of two restricted stacks in series, ruled by a right-greedy procedure and the stacks avoid some specified patterns. Some of the obtained results have been further generalized to Cayley permutations by Cerbai, specialized to particular patterns by Defant and Zheng, or considered in the context of functions over the symmetric group by Berlow. In this work we study pattern avoiding machines where the first stack avoids a pair of patterns of length 3 and investigate those pairs for which sortable permutations are counted by the (binomial transform of the) Catalan numbers and the Schröder numbers.
\end{abstract}

(c) 2021 Published by Elsevier B.V.

\section{Introduction}

Pattern avoiding machines were recently introduced in [7] in attempt to gain a better understanding of sortable permutations using stacks in series. They consist of two restricted stacks in series, equipped with a right-greedy procedure, where the first stack avoids a fixed pattern, reading the elements from top to bottom; and the second stack avoids the pattern 21 (which is a necessary condition for the machine to sort permutations). The authors of [7] provide a characterization of the avoided patterns for which sortable permutations do not form a class, and they show that those patterns are enumerated by the Catalan numbers. For specific patterns, such as 123 and the decreasing pattern of any length, a geometrical description of sortable

\footnotetext{
* Corresponding author.

E-mail addresses: barjl@u-bourgogne.fr (J.-L. Baril), giulio.cerbai@unifi.it (G. Cerbai), carine.khalil@u-bourgogne.fr (C. Khalil), vvajnov@u-bourgogne.fr (V. Vajnovszki).

1 G.C. is member of the INdAM Research group GNCS; he is partially supported by INdAM-GNCS 2020 project "Combinatoria delle permutazioni, delle parole e dei grafi: algoritmi e applicazioni”.
}

permutations is also obtained. The pattern 132 has been solved later in [8]. Some of these results have been further generalized to Cayley permutations in [9]. More recently, Berlow [5] explores a single stack version of pattern avoiding machines, where the stack avoids a set of patterns and the sorting process is regarded as a function. Analogous machines, but based on the notion of consecutive patterns, have been introduced and discussed in [10].

In this work we study a variant of pattern-avoiding machines where the first stack avoids $(\sigma, \tau)$, a pair of patterns of length three. Following [7], we call it $(\sigma, \tau)$ machine. More specifically, we restrict ourselves to those pairs of patterns for which sortable permutations are counted by either the Catalan numbers or two of their close relatives: the binomial transform of Catalan numbers and the Schröder numbers. For the pair $(132,231)$ we show that sortable permutations are those avoiding 1324 and 2314 , a set whose enumeration is given by the large Schröder numbers. Under certain conditions on the avoided patterns, the output of the first stack is bijectively related to its input (see [5,9]): it follows that for three pairs of patterns, namely $(123,213),(132,312)$ and $(231,321)$, sortable permutations are counted by the Cata- 
lan numbers. This result was conjectured in [3] and settled independently in $[4,5]$. For the pair $(123,132)$, we prove that sortable permutations are those avoiding the patterns 2314, 3214, 4213 and the generalized pattern [2413. We prove that sortable permutations are enumerated by the Catalan numbers by showing that the distribution of the first element is given by the well-known Catalan triangle. Finally, we show that for the pair $(123,312)$ the corresponding counting sequence is the binomial transform of Catalan numbers.

This paper is the extended version of the conference presentation [4] and some of the presented results previously appear in [3].

\section{Notations and some preliminary results}

We start by recalling some classical definitions about pattern avoidance on permutations (see [12] for a more detailed introduction). Denote by $\mathfrak{S}_{n}$ the set of permutations of length $n$ and let $\mathfrak{S}=\cup_{n \geq 0} \mathfrak{S}_{n}$. Given two permutations $\sigma$ of length $k$ and $\pi=\pi_{1} \cdots \pi_{n}$, we say that $\pi$ contains the pattern $\sigma$ if $\pi$ contains a subsequence $\pi_{i_{1}} \cdots \pi_{i_{k}}$, with $i_{1}<i_{2}<\cdots<i_{k}$, which is order isomorphic to $\sigma$. In this case, we say that $\pi_{i_{1}} \cdots \pi_{i_{k}}$ is an occurrence of the pattern $\sigma$ in $\pi$. Otherwise, we say that $\pi$ avoids $\sigma$.

We say that $\pi$ contains an occurrence of the (generalized) pattern $[\sigma$ if $\pi$ contains an occurrence of $\sigma$ that involves the first element $\pi_{1}$ of $\pi$. For instance, an occurrence of [12 in $\pi$ corresponds to a pair of elements $\pi_{1} \pi_{i}$, with $i>1$ and $\pi_{i}>\pi_{1}$. A barred pattern $\tilde{\sigma}$ is a pattern where some entries are barred. Let $\sigma$ be the classical pattern obtained by removing all the bars from $\tilde{\sigma}$. Let $\tau$ be the pattern which is order isomorphic to the non-barred entries of $\tilde{\sigma}$ (i.e. obtained from $\tilde{\sigma}$ by removing all the barred entries and suitably rescaling the remaining elements). A permutation $\pi$ avoids $\tilde{\sigma}$ if each occurrence of $\tau$ in $\pi$ can be extended to an occurrence of $\sigma$. For instance, a permutation $\pi$ avoids the pattern [241̄3 if for any subsequence $\pi_{1} \pi_{i} \pi_{j}$, with $1<i<j$ and $\pi_{1}<\pi_{j}<\pi_{i}$, there is an index $t, i<t<j$, such that $\pi_{1} \pi_{i} \pi_{t} \pi_{j}$ is an occurrence of 2413.

Given a set of (generalized) patterns $T$, denote by $A v_{n}(T)$ the set of permutations in $\mathfrak{S}_{n}$ avoiding each pattern in $T$. Similarly, let $\operatorname{Av}(T)=\cup_{n \geq 0} \operatorname{Av}_{n}(T)$. If $T=\{\sigma\}$ is a singleton, we write $\operatorname{Av}_{n}(\sigma)$ and $\operatorname{Av}(\sigma)$. In his celebrated book [13], Knuth gave the following characterization of stack sortable permutations, which is often considered the starting point of stack sorting and permutation patterns disciplines.

Proposition 1 ([13]). A permutation $\pi$ is sortable using a classical stack (that is, a 21-avoiding stack) if and only if $\pi$ avoids the pattern 231.

Let $T$ be a set of patterns. A $T$-stack is a stack that is not allowed to contain an occurrence of any pattern in $T$, reading its elements from top to bottom. Given a permutation $\pi$, denote by out ${ }^{T}(\pi)$ the permutation obtained after passing $\pi$ through the $T$-avoiding stack by applying a greedy procedure, i.e. by always pushing the next element of the input, unless it creates an occurrence of a forbidden pattern inside the stack. Denote by $\operatorname{Sort}_{n}(T)$ the set of length $n$ permutations that are sortable by the $T$-machine, that is, by passing $\pi$ through the $T$-avoiding stack and then through the 21-avoiding stack. Permutations in $\operatorname{Sort}_{n}(T)$ are called $T$-sortable, and $\operatorname{Sort}(T)$ is the set of $T$-sortable permutations of any length. As a consequence of Proposition 1, Sort $(T)$ consists precisely of those permutations $\pi$ for which $\operatorname{out}^{T}(\pi)$ avoids 231 . To ease notations, if $T$ is either a singleton $T=\{\sigma\}$ or a pair of patterns $T=\{\sigma, \tau\}$, we will omit the curly brackets from the above notations. For instance, we will write $\operatorname{Sort}(\sigma, \tau)$ instead of $\operatorname{Sort}(\{\sigma, \tau\})$.

The authors of [7] showed that if $\pi$ is a 12-sortable permutation of length $n$, then $\operatorname{out}^{12}(\pi)=n(n-1) \cdots 1$. Moreover, by Proposition 1 and applying the complement operation on the processed permutation, we have that Sort(12) $=A v(213)$. In order to refer to this result later, we state it below in a slightly more general form. A partial permutation of $n$ is an injection $\pi:\{1,2, \ldots, k\} \rightarrow$ $\{1,2, \ldots, n\}$, for some $0 \leq k \leq n$, and the integer $k$ is said to be the length of $\pi$. We let a 12-stack act on a partial permutation $\pi$ of $n$ in the natural way by identifying $\pi$ with the list of its images.

Proposition 2. If $\pi$ is a partial permutation of $n$ which is 12sortable, then out ${ }^{12}(\pi)$ is the decreasing rearrangement of the symbols of $\pi$. Moreover, $\pi$ is 12-sortable if and only if it avoids 213.

An entry $\pi_{i}$ of a permutation $\pi$ is a left-to-right minimum if $\pi_{i}<\pi_{j}$, for each $j<i$. The left-to-right minima decomposition (briefly ltr-min decomposition) of $\pi$ is $\pi=$ $m_{1} B_{1} m_{2} B_{2} \cdots m_{t} B_{t}$, where $m_{1}>m_{2}>\cdots>m_{t}$ are the ltrminima of $\pi$ and the block $B_{i}$ contains the elements of $\pi$ between $m_{i}$ and $m_{i+1}$, for $i=1, \ldots, t-1$. The last block $B_{t}$ contains the elements that follow $m_{t}$ in $\pi$. Note that $m_{t}=1$. The notion of left-to-right maximum of a permutation $\pi$ is defined similarly. The ltr-max decomposition of $\pi$ is $\pi=M_{1} B_{1} M_{2} B_{2} \cdots M_{t} B_{t}$, where $M_{1}<M_{2}<\cdots<M_{t}$ are the ltr-maxima of $\pi$. In this case $M_{t}=n$, where $n$ is the length of $\pi$.

Finally, the sequence $\left(c_{n}\right)_{n \geq 0}$, pervasive in this paper, is the sequence of Catalan numbers $c_{n}=\frac{1}{n+1}\left(\begin{array}{c}2 n \\ n\end{array}\right)(\mathrm{A} 000108$ in [15]).

\section{Pair $(132,231)$}

This section is devoted to the analysis of the $(123,231)$ machine.

Theorem 1. Consider the $(132, \sigma)$-machine, where $\sigma=\sigma_{1} \cdots$ $\sigma_{k-1} \sigma_{k} \in \mathfrak{S}_{k}$, with $k \geq 3$ and $\sigma_{k-1}>\sigma_{k}$. Given a permutation $\pi$ of length $n$, let $m_{1} B_{1} \cdots m_{t} B_{t}=\pi$ be its ltr-min decomposition. Then:

1. Every time a ltr-minimum $m_{i}$ is pushed into the $(132, \sigma)$ stack, the $(132, \sigma)$-stack contains the elements $m_{i-1}, \ldots$, $m_{2}, m_{1}$, reading from top to bottom. Moreover, we have

$$
\operatorname{out}^{132, \sigma}(\pi)=\tilde{B}_{1} \cdots \tilde{B}_{t} m_{t} \cdots m_{1}
$$


where $\tilde{B}_{i}$ is a rearrangement of $B_{i}$.

2. If $\pi$ is $(132, \sigma)$-sortable, then $\tilde{B}_{i}$ is decreasing for each $i$. Moreover, for each $i \leq t-1$, we have $B_{i}>B_{i+1}$ (i.e. $x>y$ for each $x \in B_{i}, y \in \bar{B}_{i+1}$ ).

Proof. 1. Let us consider the evolution of the $(132, \sigma)$ stack on input $\pi$. Note that, since $k \geq 3$, the element $m_{1}$ remains at the bottom of the $(132, \sigma)$-stack until the end of the process. Now, if $B_{1}$ is not empty then for each $x \in B_{1}$, the elements $m_{2} x m_{1}$ form an occurrence of 132 . Therefore the block $B_{1}$ is extracted before $m_{2}$ enters the $(132, \sigma)$-stack. After $m_{2}$ is pushed, the $(132, \sigma)$-stack contains $m_{2} m_{1}$, reading from top to bottom. Since $m_{2}<m_{1}$, but $\sigma_{k-1}>\sigma_{k}$ by hypothesis, $m_{2}$ cannot play the role of either $\sigma_{k-1}$ in an occurrence of $\sigma$ or of 3 in an occurrence of 132. Thus $m_{2}$ remains at the bottom of the $(132, \sigma)$-stack until the end of the sorting procedure. The thesis follows by iterating the same argument on each block $B_{i}$, for $i \geq 2$.

2. Suppose that $\pi$ is $(132, \sigma)$-sortable. Assume, for a contradiction, that $\tilde{B}_{i}$ is not decreasing, for some $i$. Then there are two consecutive elements $x<y$ in $\tilde{B}_{i}$. Therefore, by what proved above, out ${ }^{132, \sigma}(\pi)$ contains an occurrence $x y m_{t}$ of 231, which is impossible due to Proposition 1. Finally, suppose that $x<y$, for $x \in B_{i}$ and $y \in B_{i+1}$. Then $x y m_{t}$ is an occurrence of 231 in out $^{132, \sigma}(\pi)$, a contradiction.

Theorem 1 and Proposition 2 guarantee that if $\pi=$ $m_{1} B_{1} \cdots m_{t} B_{t}$ is the ltr-min decomposition of a $(132,231)-$ sortable permutation $\pi$, then (with the notation above) $\tilde{B}_{i}=$ out $^{12}\left(B_{i}\right)$, for each $i$. However, this is true even when the sortability requirement is relaxed.

Lemma 1. Let $\pi=m_{1} B_{1} \cdots m_{t} B_{t}$ be the ltr-min decomposition of a permutation $\pi$. Write out ${ }^{132,231}(\pi)=\tilde{B}_{1} \cdots \tilde{B}_{t} m_{t} \cdots m_{1}$ as in Theorem 1. Then $\tilde{B}_{i}=$ out $^{12}\left(B_{i}\right)$, for each $i$.

Proof. Consider the instant immediately after $m_{i}$ is pushed into the $(132,231)$-stack and the non-empty block $B_{i}$ has to be processed, for some $i$. By Theorem 1, at this point the $(132,231)$-stack contains $m_{i}, m_{i-1}, \ldots, m_{1}$, reading from top to bottom. We want to show that the behavior of the (132, 231)-stack on $B_{i}$ is equivalent to the behavior of an empty 12 -stack on input $B_{i}$. We prove that the $(132,231)$ stack performs the pop operation of some $x \in B_{i}$ if and only if the 12-stack does the same. If either the next element of the input is $m_{i+1}$ or $x$ is the last element of $\pi$ to be processed, then both the $(132,231)$-stack and the 12-stack perform a pop operation, as desired. Otherwise, suppose the next element of the input is $y$, for some $y$ in the same block $B_{i}$, and the $(132,231)$-stack pops the element $x \in B_{i}$. This means that the $(132,231)$-stack contains two elements $z, w$, with $z$ above $w$, such that $y z w$ is an occurrence of either 132 or 231 . Note that, since $z>w$, $z$ is not a ltr-minimum. Therefore $y z$ is an occurrence of 12 and the 12-stack performs a pop operation, as desired. Conversely, suppose that the 12-stack pops the element $x$, with $y \in B_{i}$ the next element of the input. This implies that the 12-stack contains an element $z$ such that $z>y$.
Therefore $y z m_{i}$ is an occurrence of 231 and the $(132,231)$ stack performs a pop operation, as desired.

Corollary 1 . Let $\pi=m_{1} B_{1} \cdots m_{t} B_{t}$ be the ltr-min decomposition of a permutation $\pi$. Then the following are equivalent.

1. $B_{i}$ avoids 213 and $B_{i}>B_{i+1}$, for each $i$.

2. $\pi$ is $(132,231)$-sortable.

3. $\pi \in \operatorname{Av}(1324,2314)$.

Proof. Combining the first point in Theorem 1 and Lemma 1 we have:

$\operatorname{out}^{132,231}(\pi)=\operatorname{out}^{12}\left(B_{1}\right) \cdots$ out $^{12}\left(B_{t}\right) m_{t} \cdots m_{1}$.

We will use this decomposition of $\operatorname{out}^{132,231}(\pi)$ throughout the rest of the proof.

$[1 \Rightarrow 2]$ Suppose, for a contradiction, that out ${ }^{132,231}(\pi)$ contains an occurrence bca of 231 . Note that, since $c>a$, while $m_{t}<\cdots<m_{1}, c$ is not a ltr-minimum of $\pi$ (and thus neither is $b$ ). Now, if $b$ and $c$ are in the same block $B_{j}$, then out ${ }^{12}\left(B_{j}\right)$ is not decreasing. Thus, by Proposition $2, B_{j}$ contains 213 , which is a contradiction. Otherwise, if $b \in B_{j}$ and $c \in B_{k}$, with $j<k$, then we have a contradiction with the hypothesis $B_{i}>B_{i+1}$ for each $i$.

$[2 \Rightarrow 3]$ Suppose, for a contradiction, that $\pi \notin \operatorname{Av}(1324$, 2314). First, suppose that $\pi$ contains an occurrence acbd of 1324. Observe that $b, c, d$ are not ltr-minima of $\pi$. Now, if $b$ and $d$ are in the same block $B_{j}$ of $\pi$, for some $j$, then $B_{j}$ contains an occurrence $c b d$ of 213 . Therefore out ${ }^{12}\left(B_{j}\right)$ contains an occurrence of 231 due to Proposition 2, which contradicts the hypothesis. Otherwise, if $b \in B_{j}$ and $d \in B_{k}$, for some $j<k$, then out ${ }^{132,231}(\pi)$ contains an occurrence $b d m_{k}$ of 231 , again a contradiction. The pattern 2314 can be addressed analogously, so we leave it to the reader.

[ $3 \Rightarrow 1$ ] Let $\pi \in \operatorname{Av}(1324,2314)$. If $B_{i}$ contains an occurrence bac of 213 , then $\pi$ contains an occurrence $m_{i}$ bac of 1324 , which is impossible. Otherwise, if $\pi$ contains two elements $x \in B_{j}, y \in B_{k}$, with $x<y$ and $j<k$, then $m_{j} x m_{k} y$ is an occurrence of 2314 , contradicting the hypothesis.

The enumeration of $\operatorname{Av}(1324,2314)$ (or a symmetry of these patterns) can be found for instance in $[2,16]$. Note that in [1], the authors provide a constructive bijection between these permutations and Schröder paths.

Corollary 2. Permutations of length $n$ in Sort $(132,231)$ are enumerated by the large Schröder numbers (sequence A0 06318 in [15]).

\section{The $(\sigma, \hat{\sigma})$-machine}

For a permutation $\sigma$ of length two or more, denote by $\hat{\sigma}$ the permutation obtained from $\sigma$ by interchanging its first two entries. Let us regard a $(\sigma, \tau)$-stack as an operator out ${ }^{\sigma, \tau}: \mathfrak{S} \rightarrow \mathfrak{S}$. By conveniently modifying the proof of Corollary 4.5 in [9] (stated in the context of Cayley permutations), we have that out ${ }^{\sigma, \tau}$ is a length preserving bijection on $\mathfrak{S}$ if and only if $\tau=\hat{\sigma}$. More generally, Berlow [5] showed that for a set $T$ of patterns, out ${ }^{T}$ is a 
length preserving bijection on $\mathfrak{S}$ if and only if $T$ is closed under the ${ }^{\wedge}$ operator. In order for the paper to be selfcontained, we shall give the following result, which is easier to prove (although weaker): out ${ }^{\sigma, \hat{\sigma}}$ is a bijection for any pattern $\sigma$. An immediate consequence will be Theorem 2 below.

Let $N^{*}$ be the set of finite length integer sequences. The action of the $(\sigma, \tau)$-stack on input $\pi$ can be naturally represented as a sequence of triples $(r ; s ; t) \in\left(N^{*}\right)^{3}$, where $r$ is the current content of the output, $s$ is the current content of the $(\sigma, \tau)$-stack (read from top to bottom) and $t$ is the current content of the input. The triple $(r ; s ; t)$ is said to be a state of passing of $\pi$ through the $(\sigma, \tau)$-stack. Clearly, $r$ is a prefix of out ${ }^{\sigma, \tau}(\pi), t$ is a suffix of $\pi$, the initial state is $(\lambda ; \lambda ; \pi)$ and the final one is $\left(\right.$ out $\left.^{\sigma, \tau}(\pi) ; \lambda ; \lambda\right)$, where $\lambda$ is the empty sequence. Moreover a non-final state $\left(p_{1} p_{2} \cdots p_{a} ; s_{1} s_{2} \cdots s_{b} ; t_{1} t_{2} \cdots t_{c}\right)$ is followed by either the state

$\left(p_{1} p_{2} \cdots p_{a} s_{1} ; s_{2} \cdots s_{b} ; t_{1} t_{2} \cdots t_{c}\right)$,

if a pop operation is performed next, or

$\left(p_{1} p_{2} \cdots p_{a} ; t_{1} s_{1} s_{2} \cdots s_{b} ; t_{2} \cdots t_{c}\right)$,

if a push operation is performed next.

For $p=p_{1} \cdots p_{n} \in \mathbb{N}^{n}$, we denote by $p^{r}$ the reverse of $p$, that is $p^{\mathrm{r}}=p_{n} \cdots p_{1}$. We wish to show that the behavior of the $(\sigma, \hat{\sigma})$-stack on $\pi$ is strictly related to its behavior on $\left(\text { out }^{\sigma, \hat{\sigma}}(\pi)\right)^{\mathrm{r}}$. More precisely, if $o_{1} \cdots o_{2 n}$ is the sequence of push/pop operations performed when $\pi$ is passed through a $(\sigma, \hat{\sigma})$-stack, then $o_{2 n}^{\prime} \cdots o_{1}^{\prime}$ is the sequence of push/pop operations performed when $\left(\text { out }^{\sigma, \hat{\sigma}}(\pi)\right)^{\mathrm{r}}$ is passed through the $(\sigma, \hat{\sigma})$-stack, where $o_{i}^{\prime}$ is a push (resp. pop) operation if $o_{i}$ is a pop (resp. push) operation. This can be equivalently expressed by saying that the state $(p ; s ; t)$ is followed by $(u ; v ; w)$ if and only if the state $\left(w^{\mathrm{r}} ; v ; u^{\mathrm{r}}\right)$ is followed by $\left(t^{\mathrm{r}} ; s ; p^{\mathrm{r}}\right)$.

Lemma 2. Consider the action of the $(\sigma, \hat{\sigma})$-stack. Let $p, s, t \in$ $\mathbb{N}^{*}$ and $x \in \mathbb{N}$.

1. If the state $(p, x s, t)$ is followed by the state $(p x, s, t)$ (and thus a pop operation is performed) then the state $\left(t^{\mathrm{r}}, s, x p^{\mathrm{r}}\right)$ is followed by the state $\left(t^{\mathrm{r}}, x s, p^{\mathrm{r}}\right)$ (and thus a push operation is performed).

2. If the state $(p, s, x t)$ is followed by the state $(p, x s, t)$ (and thus a push operation is performed), then the state $\left(t^{\mathrm{r}}, x s, p^{\mathrm{r}}\right)$ is followed by the state $\left(t^{\mathrm{r}} x, s, p^{\mathrm{r}}\right)$ (and thus a pop operation is performed).

Proof. 1. Since $x s$ is the content of the $(\sigma, \hat{\sigma})$-stack in the state $(p, x s, t)$, we have that $x s$ avoids $\sigma$ and $\hat{\sigma}$. Thus a push operation is performed if $s$ is the content of the $(\sigma, \hat{\sigma})$-stack and $x$ is the next element of the input.

2. If $p$ is empty, the statement holds. Otherwise, let $p=$ $p_{1} \cdots p_{a}$ and $s=s_{1} \cdots s_{b}$. Observe that $p_{a}$ is the last element that has been extracted from the $(\sigma, \hat{\sigma})$-stack before $x$ enters. Therefore, when $p_{a}$ is extracted, $p_{a}$ plays the role of either $\sigma_{2}$ in an occurrence of $\sigma$ or of $\hat{\sigma}_{2}$ in an occurrence of $\hat{\sigma}$. More precisely, one of the following four cases hold. We show the details for the first case only, the others being similar. Let $z$ be the length of $\sigma$.

- $s_{\ell} p_{a} s_{i_{3}} \cdots s_{i_{z}}$ is an occurrence of $\sigma$, for some $\ell \geq 1$ and $\ell<i_{3}<\cdots<i_{z}$. Then $p_{a} s_{\ell} s_{i_{3}} \cdots s_{i_{z}}$ is an occurrence of $\hat{\sigma}$ and therefore a pop operation is performed when $p_{a}$ is the next element of the input and $x s$ is the content of the $(\sigma, \hat{\sigma})$-stack, as desired.

- $s_{\ell} p_{a} s_{i_{3}} \cdots s_{i_{z}}$ is an occurrence of $\hat{\sigma}$, for some $\ell \geq 1$ and $\ell<i_{3}<\cdots<i_{z}$.

- $x p_{a} s_{i_{3}} \cdots s_{i_{z}}$ is an occurrence of $\sigma$, for some $i_{3}<$ $\cdots<i_{z}$.

- $x p_{a} s_{i_{3}} \cdots s_{i_{z}}$ is an occurrence of $\hat{\sigma}$, for some $i_{3}<$ $\cdots<i_{z}$

A straightforward consequence of the previous lemma is that the map $\left(\text { out }^{\sigma, \hat{\sigma}}\right)^{\mathrm{r}}: \mathfrak{S} \rightarrow \mathfrak{S}$ is its own inverse, and thus a bijection. More specifically, for any permutation $\pi$, we have $\left(\text { out }^{\sigma, \hat{\sigma}}\right)^{-1}(\pi)=\left(\operatorname{out}^{\sigma, \hat{\sigma}}\left(\pi^{\mathrm{r}}\right)\right)^{\mathrm{r}}$. Since $\pi$ is $(\sigma, \hat{\sigma})$-sortable if and only if out ${ }^{\sigma, \hat{\sigma}}(\pi)$ avoids 231 (and the reverse map is bijective), we have that $\operatorname{Sort}(\sigma, \hat{\sigma})$ is in bijection with $\operatorname{Av}(231)$. The next theorem follows.

Theorem 2. For any pattern $\sigma$, out $t_{n}^{\sigma, \hat{\sigma}}$ is a bijection on $\mathfrak{S}_{n}$. Moreover, we have

$$
\begin{aligned}
\left|\operatorname{Sort}_{n}(123,213)\right| & =\left|\operatorname{Sort}_{n}(132,312)\right| \\
& =\left|\operatorname{Sort}_{n}(231,321)\right|=c_{n},
\end{aligned}
$$

the $n$th Catalan number.

\section{Pair $(123,132)$}

We characterize Sort $(123,132)$ in terms of pattern avoidance. Then we show that $(123,132)$-sortable permutations are enumerated by the Catalan numbers by exhibiting a link with the very well studied Catalan triangle.

Theorem 3. A permutation $\pi$ is $(123,132)$-sortable if and only if $\pi$ avoids 2314, 3214, 4213 and [2413.

Proof. Suppose that $\pi$ is $(123,132)$-sortable. For a contradiction, suppose that $\pi$ contains $\tau \in\{2314,3214,4213\}$. Pick an occurrence $\pi_{i} \pi_{j} \pi_{k} \pi_{\ell}$ of $\tau$, with $i<j<k<\ell$, where $\ell$ is chosen minimal, and $k, j$, and $i$ are chosen maximal, in this order.

If $\tau=2314$, due to our choice of $i, j, k, \ell$, we have $\pi_{i}<$ $\pi_{u}<\pi_{j}$, for $k<u<\ell$. Now, when $\pi_{k}$ is pushed in the $(123,132)$-stack, at least one of $\pi_{i}$ and $\pi_{j}$ has already been extracted: otherwise the $(123,132)$-stack would contain an occurrence of 132, which is forbidden. For each $u$, $k+1 \leq u \leq \ell$, we have $\pi_{k}<\pi_{u}$ and when $\pi_{u}$ is pushed in the $(123,132)$-stack, $\pi_{k}$ is still in the $(123,132)$-stack. Indeed, $\pi_{u} \pi_{x} \pi_{y}$ cannot be an occurrence of 123 nor of 132 with $\pi_{x}$ above $\pi_{y}$, both in the tail of the $(123,132)$-stack 
beginning by $\pi_{k}$. If $\pi_{i}$ (resp. $\pi_{j}$ ) is extracted before $\pi_{k}$ enters in the $(123,132)$-stack, then $\pi_{i} \pi_{k+1} \pi_{k}\left(\operatorname{resp} . \pi_{j} \pi_{\ell} \pi_{k}\right)$ creates a pattern 231 in out ${ }^{123,132}(\pi)$, a contradiction.

If $\tau=3214$, due to our choice of $i, j, k, \ell$, we have $\pi_{i}>$ $\pi_{u}>\pi_{j}$, for $k<u<\ell$; and $\pi_{u}<\pi_{u+1}$, for $k \leq u<\ell$. As above, when $\pi_{k}$ is pushed in the $(123,132)$-stack, at least one of $\pi_{i}$ and $\pi_{j}$ has already been extracted: otherwise the $(123,132)$-stack would contain an occurrence of 123 . Since $\pi_{k+1}>\pi_{k}$, the next step pushes $\pi_{k+1}$ in the $(123,132)$-stack. ( $i)$ Assume that $\pi_{i}$ and $\pi_{j}$ both had left the $(123,132)$-stack. Then $\pi_{k}$ is just below $\pi_{k+1}$ in the $(123,132)$-stack, and $\pi_{k}<\pi_{j}<\pi_{k+1}$. This implies that $\pi_{j} \pi_{k+1} \pi_{k}$ is an occurrence of 231 in out ${ }^{123,132}(\pi)$, a contradiction. (ii) Assume that $\pi_{i}$ is still in the $(123,132)$ stack and $\pi_{j}$ had left this stack. Again, $\pi_{j} \pi_{k+1} \pi_{k}$ is an occurrence of 231 in out ${ }^{123,132}(\pi)$, a contradiction. (iii) Assume that $\pi_{j}$ is still in the $(123,132)$-stack and $\pi_{i}$ had left this stack. Since $\pi_{u}<\pi_{u+1}$ for $k \leq u \leq \ell-1$, the next steps of the process push successively all entries $\pi_{k+1}, \ldots, \pi_{\ell}$ in the $(123,132)$-stack. As above, $\pi_{i} \pi_{\ell} \pi_{k}$ is an occurrence of 231 in out ${ }^{123,132}(\pi)$, again a contradiction.

The case $\tau=4213$ can be treated similarly.

Finally, suppose that $\pi$ contains [241̄3. Equivalently, there are two indices $i<j$ such that $\pi_{1} \pi_{i} \pi_{j}$ is an occurrence of 132 and $\pi_{k}>\pi_{1}$ for each $i<k<j$. Observe that, by choosing $j$ minimal and $i$ maximal (in this order), we can assume $j=i+1$. Now, if $\pi_{i}$ is still in the $(123,132)$ stack when $\pi_{i+1}$ enters, then out ${ }^{123,132}(\pi)$ contains an occurrence $\pi_{i+1} \pi_{i} \pi_{1}$ of 231 , which is impossible due to the sortability of $\pi$. Therefore $\pi_{i}$ is extracted before $\pi_{i+1}$ enters. This means that there are two elements $\pi_{u}, \pi_{v}$ in the $(123,132)$-stack, with $u<v$ (and thus $\pi_{v}$ above $\pi_{u}$ ), such that $\pi_{i+1} \pi_{v} \pi_{u}$ is an occurrence of either 123 or 132 . Choose $u, v$ minimal amongst those indices, ${ }^{2}$ so that $\pi_{u}$ is still in the $(123,132)$-stack when $\pi_{i+1}$ enters. Notice that $\pi_{i+1}<\pi_{u}$ (and thus $u \neq 1$ ). Moreover, it must be $\pi_{u}<\pi_{i}$, otherwise $\pi_{i} \pi_{u} \pi_{1}$ would be an occurrence of 231 in the $(123,132)$-stack, which is forbidden. But then $\pi_{i+1} \pi_{u} \pi_{1}$ is an occurrence of 231 in out ${ }^{123,132}(\pi)$, a contradiction.

Conversely, suppose that $\pi$ is not $(123,132)$-sortable. We shall prove that $\pi$ contains at least one of the patterns $3214,2314,4213$ or $\left[24 \overline{1} 3\right.$. By hypothesis out ${ }^{123,132}(\pi)$ contains an occurrence bca of 231. Let $b=\pi_{j}$ and $c=\pi_{k}$, for some indices $j, k$. We distinguish two cases, according whether $j<k$ or $j>k$.

- Suppose that $j<k$ and thus $\pi_{j}$ is extracted from the $(123,132)$-stack before $\pi_{k}$ enters. Then there are two elements $\pi_{u}, \pi_{v}$ in the $(123,132)$-stack, with $u<v$ (and thus $\pi_{v}$ above $\pi_{u}$ ), such that $\pi_{z} \pi_{v} \pi_{u}$ is an occurrence of either 123 or 132 , where $\pi_{z}$ is the next element of the input. Notice that $\pi_{j} \geq \min \left\{\pi_{u}, \pi_{v}\right\}$, since otherwise $\pi_{j} \pi_{v} \pi_{u}$ would be an occurrence of either 123 or 132 in the $(123,132)$-stack, which is impossible. Thus $\pi_{k}>\pi_{j}>\pi_{z}$. If $\pi_{z} \pi_{v} \pi_{u}$ is an occurrence of 123 , then $\pi_{u} \pi_{v} \pi_{z} \pi_{k}$ is an occurrence of

\footnotetext{
2 In other words, pick the deepest such elements $\pi_{u}, \pi_{v}$ in the $(123,132)$-stack.
}

either 4213, if $\pi_{u}>\pi_{k}$, or 3214, if $\pi_{u}<\pi_{k}$. Finally, if $\pi_{z} \pi_{v} \pi_{u}$ is an occurrence of 132 , then $\pi_{u} \pi_{j} \pi_{z} \pi_{k}$ is an occurrence of 2314 .

- Suppose instead that $j>k$ and thus $\pi_{k}$ is still in the $(123,132)$-stack when $\pi_{j}$ enters. Observe that $k \neq 1$, since $\pi_{1}$ is the last element of out ${ }^{123,132}(\pi)$. Therefore, when $\pi_{j}$ enters the $(123,132)$-stack, the (123, 132)-stack contains the elements $\pi_{j} \pi_{k} \pi_{1}$, reading from top to bottom. Notice that $\pi_{j}>\pi_{1}$, otherwise $\pi_{j} \pi_{k} \pi_{1}$ would realize an occurrence of the forbidden 132 inside the $(123,132)$-stack. Moreover, for each entry $\pi_{t}$, with $k<t<j$, we have $\pi_{t}>\pi_{1}$. Otherwise $\pi_{t} \pi_{k} \pi_{1}$ would be an occurrence of 132 and $\pi_{k}$ would be extracted before $\pi_{j}$, which is impossible due to our assumptions. Thus $\pi_{1} \pi_{k} \pi_{j}$ is an occurrence of [24 $\overline{1} 3$. This completes the proof.

Proposition 3. The distribution of the first element in Sort(123, 132) is given by the Catalan triangle (sequence A009766 in [15]).

Proof. Let $A_{n}(k)$ be the set of $(123,132)$-sortable permutations of length $n$ and starting with $k$. Let $A_{n}^{1}(k)$ be the subset of $A_{n}(k)$ consisting of those permutations $\pi=$ $\pi_{1} \pi_{2} \ldots \pi_{n}$ where any occurrence $\pi_{1} \pi_{i} \pi_{\ell}$ of [231 with $\pi_{\ell}=\pi_{1}-1$ can be extended into an occurrence $\pi_{1} \pi_{i} \pi_{j} \pi_{\ell}$ of [3412. Set $A_{n}^{2}(k)=A_{n}(k) \backslash A_{n}^{1}(k)$ and let $k \geq 2$. We shall provide bijections $\alpha: A_{n}^{1}(k) \rightarrow A_{n}(k-1)$ and $\beta: A_{n}^{2}(k) \rightarrow$ $A_{n-1}(k)$

Define $\alpha: A_{n}^{1}(k) \rightarrow A_{n}(k-1)$ by $\alpha(\pi)=\pi^{\prime}$, where $\pi^{\prime}$ is obtained from $\pi$ by swapping the two entries $\pi_{1}$ and $\pi_{1}-1$ in $\pi$. Since $\pi \in A_{n}^{1}(k)$, it is easy to check that $\pi^{\prime}$ avoids [2413. In addition, swapping $\pi_{1}$ and $\pi_{1}-1$ does not affect the avoidance of the three patterns 3214, 2314, 4213, which implies (see Theorem 3) that $\alpha(\pi) \in A_{n}(k-$ $1)$.

Next define $\beta: A_{n}^{2}(k) \rightarrow A_{n-1}(k)$ by $\beta(\pi)=\pi^{\prime \prime}$, where $\pi^{\prime \prime}$ is obtained from $\pi$ by deleting the entry $\pi_{\ell}$ immediately before $k-1$ and by decreasing by one all entries of $\pi$ greater than $\pi_{\ell}$. Notice that $\beta(\pi) \in A_{n-1}(k)$. Let us now sketch the proof that $\beta$ is bijective, leaving some technical details to the reader. We shall explicitly define the inverse map of $\beta$. Given $\pi \in A_{n-1}(k)$, choose an integer $\ell$ as follows:

- $\ell$ is the minimal entry $l=\pi_{u}>\pi_{1}$, with $1<u<i$, such that there is an index $v$ with $\pi_{v}<\pi_{i}$ and $u<$ $v<i$, if such entry exists.

- Otherwise, set $\ell=n$.

The preimage $\pi$ is obtained by inserting $\ell$ immediately before $\pi_{i}=k-1$ and then increasing by one all the entries $\pi_{j}$ of $\pi$ with $\pi_{j} \geq \ell$.

Finally, setting $a_{n}^{k}=\left|A_{n}(k)\right|$, we have that $a_{n}^{k}=a_{n}^{k-1}+$ $a_{n-1}^{k}$, for $2 \leq k \leq n$. Since $A_{n}(1)=\{123 \cdots n\}$ and $A_{n}(n)$ is the set of length $n$ permutations avoiding 213 and starting with $n$, the initial conditions are given by $a_{n}^{1}=1$ and $a_{n}^{n}=c_{n-1}$, where $c_{n}$ is the $n$th Catalan number. Therefore, $a_{n}^{k}$ generates the well-known Catalan triangle (see Table 1 and $[6,11,14])$. 
Table 1

The Catalan triangle $a_{n}^{k}=\left|A_{n}(k)\right|$, with $1 \leq n \leq 8$ and $1 \leq k \leq 6$.

\begin{tabular}{c|llllllll}
\hline$k \backslash n$ & 1 & 2 & 3 & 4 & 5 & 6 & 7 & 8 \\
\hline 1 & 1 & 1 & 1 & 1 & 1 & 1 & 1 & 1 \\
2 & & 1 & 2 & 3 & 4 & 5 & 6 & 7 \\
3 & & & 2 & 5 & 9 & 14 & 20 & 27 \\
4 & & & & 5 & 14 & 28 & 48 & 75 \\
5 & & & & & 14 & 42 & 90 & 165 \\
6 & & & & & & 42 & 132 & 297 \\
$\ldots$ & & & & & & & $\ldots$ & $\ldots$ \\
$\sum$ & 1 & 2 & 5 & 14 & 42 & 132 & 429 & 1430 \\
\hline
\end{tabular}

Corollary 3. Permutations of length $n$ in Sort $(123,132)$ are enumerated by the Catalan numbers.

Proof. With the previous notations we have $\mid \operatorname{Sort}_{n}(123$, $132) \mid=\sum_{k=1}^{n} a_{n}^{k}=c_{n}$, the $n$th Catalan number (see again $[6,11,14])$.

\section{Pair $(123,312)$}

We start by giving a ltr-max counterpart of Theorem 1 .

Theorem 4. Consider the $(312, \sigma)$-machine, where $\sigma=\sigma_{1} \cdots$ $\sigma_{k-1} \sigma_{k} \in \mathfrak{S}_{k}$, with $k \geq 3$ and $\sigma_{k-1}<\sigma_{k}$. Given a permutation $\pi$ of length $n$, let $\pi=M_{1} B_{1} \cdots M_{t} B_{t}$ be its ltr-max decomposition. Then:

1. Everytime a ltr-maximum $M_{i}$ is pushed into the $(312, \sigma)$ stack, the $(312, \sigma)$-stack contains the elements $M_{i}, M_{i-1}$, $\ldots, M_{2}, M_{1}$, reading from top to bottom. Moreover, we have

$\operatorname{out}^{312, \sigma}(\pi)=\tilde{B}_{1} \cdots \tilde{B}_{t} M_{t} \cdots M_{1}$,

where $\tilde{B}_{i}$ is a rearrangement of $B_{i}$.

2. If $\pi$ is $(312, \sigma)$-sortable, then $M_{1}, M_{2}, \ldots, M_{t}=n-t+$ $1, n-t+2, \ldots, n$.

Proof. 1. The proof is identical to that of the first part of Theorem 1.

2. If $\pi$ is $(312, \sigma)$-sortable, then out ${ }^{312, \sigma}(\pi)$ avoids 231 by Proposition 1 . Suppose, for a contradiction, that there is an element $j \in\{n-t+1, \ldots, n\}$ which is not a ltrmaximum. Note that $j \neq \pi_{1}=M_{1}$ and $j \neq n=M_{t}$. Then, out $^{312, \sigma}(\pi)$ contains an occurrence $j n M_{1}$ of 231 , which is a contradiction.

Instantiating $\sigma$ by 123 in the previous theorem we have the next result.

Theorem 5. Let $\pi$ be a $(123,312)$-sortable permutation and let $\pi=M_{1} B_{1} \cdots M_{t} B_{t}$ be its ltr-max decomposition. Then:

1. $B_{i}$ avoids 213 for each $i$.

2. $\tilde{B}_{i}=$ out $^{12}\left(B_{i}\right)$, for each $i$.

Proof. Let $i \geq 2$. Notice that, as a consequence of Theorem 4, immediately after $M_{i}$ has been pushed in the $(123,312)$-stack, this stack contains the elements $M_{i} \ldots$ $M_{2} M_{1}$, reading from top to bottom. Moreover, these elements remain at the bottom of the $(123,312)$-stack until the end of the sorting procedure, since they are the last elements of out ${ }^{123,312}(\pi)$. This fact will be used for the rest of the proof.

1. Suppose, for a contradiction, that $B_{i}$ contains an occurrence of 213, for some $i$, and let bac be such an occurrence with $a$ 'minimal', in the sense that there is no $a^{\prime}<a$ where $b a^{\prime} c$ is an occurrence of 213 in $B_{i}$. Therefore, since $a b M_{i}$ is an occurrence of $123, b$ is extracted from the $(123,312)$-stack before $a$ enters. In addition, when $c$ enters into the $(123,312)$-stack, $a$ is still in this stack. Indeed, no entry in $B_{i}$ between $a$ and $c$ together with $a$ produces a forbidden pattern in the $(123,312)$-stack. It follows that out $^{123,312}(\pi)$ contains bca which is an occurrence of 231 , yielding a contradiction with the sortability of $\pi$.

2 . Let us consider the action of the $(123,312)$-stack on the block $B_{i}$. We wish to show that the behavior of the $(123,312)$-stack when processing $B_{i}$ is equivalent to the behavior of an empty 12-stack on input $B_{i}$. In other words, we prove that the restriction of the $(123,312)$-stack is triggered if and only if the next element of the input forms an occurrence of 12 together with some other element in the $(123,312)$-stack. Immediately after $M_{i}$ has been pushed (i.e. before the first element of $B_{i}$ is processed), the $(123,312)$-stack contains the elements $M_{i} \cdots M_{2} M_{1}$, reading from top to bottom. Observe that $B_{i}$ avoids 213 by what proved above, therefore the $(123,312)$-stack cannot be triggered by an occurrence of 312 when processing $B_{i}$. Suppose that the next element of the input $x$ forms an occurrence $x y$ of 12 with some $y \in B_{i}$. Then $x y M_{i}$ is an occurrence of 123 in the $(123,312)$-stack, and so this stack behaves as a 12-stack. Conversely, suppose that the $(123,312)$-stack is triggered by an occurrence of $x y z$ of 123 , where $x$ is the next element of the input. Since $M_{i}>M_{i-1}>\cdots>M_{1}$, necessarily $y \in B_{i}$. Thus $x y$ is an occurrence of 12 that triggers the 12 -stack, as wanted.

As a consequence of what proved so far in this section, for any $(123,312)$-sortable permutation $\pi=M_{1} B_{1} \cdots M_{t} B_{t}$ of length $n$, we have $B_{i} \in \operatorname{Av}(213)$ and $M_{1}, \ldots, M_{t}=$ $n-t+1, \ldots, n$. Moreover, by Proposition 2 , each $\tilde{B}_{i}$ in out $^{123,312}(\pi)=\tilde{B}_{1} \cdots \tilde{B}_{t} M_{t} \cdots M_{1}$ is decreasing. Therefore, for any three elements $x, y, z$, with $x \in B_{i}, y \in B_{j}$ and $z \in B_{k}$, with $i<j \leq k, x y z$ is not an occurrence of 231 . Otherwise $x y z$ would still be an occurrence of 231 in out $^{123,312}(\pi)$, contradicting the fact that $\pi$ is $(123,312)$ sortable. From now on, we say that $x y z$ is an occurrence of $2-3-1$ if $z<x<y$, with $x \in B_{i}, y \in B_{j}$ and $z \in B_{k}$, with $i<j<k$. Similarly, when $j=k$, we say that $x y z$ is an occurrence of $2-31$.

Theorem 6. Let $\pi=M_{1} B_{1} \cdots M_{t} B_{t}$ be the ltr-max decomposition of a permutation $\pi$ of length $n$. Write out ${ }^{123,312}(\pi)=$ $\tilde{B}_{1} \cdots \tilde{B}_{t} M_{t} \cdots M_{1}$ as in Theorem 4. Then $\pi$ is $(123,312)-$ sortable if and only if the following conditions are satisfied:

1. $M_{j}=n-t+j$, for each $j=1, \ldots, t$.

2. $B_{i}$ avoids 213 for each $i$ (and thus $\tilde{B}_{i}$ is decreasing for each i). 
3. out $^{123,312}(\pi)$ avoids $2-3-1$.

4. out $123,312(\pi)$ avoids $2-31$.

Proof. If $\pi$ is $(123,312)$-sortable, then $\pi$ satisfies all the above conditions as a consequence of what proved before in this section. Conversely, it is easy to check that if $\pi$ satisfies the above conditions, then out ${ }^{123,312}(\pi)$ avoids 231 . Thus $\pi$ is $(123,312)$-sortable.

Reformulating the third condition of Theorem 6 we obtain the following lemma, whose easy proof is omitted.

Lemma 3. Let $\pi=M_{1} B_{1} \cdots M_{t} B_{t}$ be the ltr-max decomposition of the $(123,312)$-sortable permutation $\pi$. Write out $^{123,312}(\pi)=\tilde{B}_{1} \cdots \tilde{B}_{t} M_{t} \cdots M_{1}$ as in Theorem 4. Then out $^{123,312}(\pi)$ avoids $2-31$ if and only if for each $x \in B_{i}$, $y \in B_{j}$, with $i<j$, we have:

- if $y>x$, then $B_{j}>x$.

- If $y<x$, then $B_{j}<x$.

In other words, Lemma 3 says that each block $B_{j}$ of a $(123,312)$-sortable permutation $\pi$ is bounded between two previous elements of $\pi$. The following result is obtained by restating this lemma and Theorem 6 in terms of pattern avoidance.

Theorem 7. A permutation $\pi$ is $(123,312)$-sortable if and only if $\pi$ avoids the three generalized patterns [132, [42531 and $[421 \overline{5} 3$.

Next we prove that $(123,312)$-sortable permutations are enumerated by the binomial transform of Catalan numbers. We shall exploit the above characterization in terms of patterns in order to provide a bijection with a certain set of partial permutations, whose enumeration is straightforward.

Recall from Section 2 that a partial permutation of $n$ is an injection $\pi:\{1,2, \ldots, k\} \rightarrow\{1,2, \ldots, n\}$, for some $0 \leq k \leq n$. The partial permutation of length zero will be denoted by $\lambda$. Denote by $\mathcal{A}_{n}$ the set of all partial permutations of $n$. For instance, we have $\mathcal{A}_{3}=\{\lambda, 1,2,3$, $12,21,13,31,23,32,123,132,213,231,312,321\}$. There is a natural bijection between the set of permutations in $\mathfrak{S}_{n}$ avoiding the pattern [132 and $\mathcal{A}_{n-1}$. Indeed, from a length $n$ permutation $\pi$ avoiding [132, we associate the unique partial permutation $\alpha(\pi) \in \mathcal{A}_{n-1}$ defined as follows:

$\alpha(\pi)_{\pi_{i}}=i-1$, for $\pi_{i}<\pi_{1}$.

In other words, $\alpha(\pi)$ is obtained by recording the indices (minus one) of the elements $\pi_{i}<\pi_{1}$, from the smallest to the largest one. For instance, if $\pi=52461783$, then $\alpha(\pi)=4172$. Notice that $\alpha(\pi)=\lambda$ if and only if $\pi_{1}=1$. Let us now define two pattern containments on $\mathcal{A}_{n}$. Let $a=a_{1} a_{2} \cdots a_{m}$ be a partial permutation of $n$, with $m \leq n$, and let $i<j<k$. Then $a_{i} a_{j} a_{k}$ is an occurrence of the pattern $31 \mid 2$ if it is an occurrence of 312 such that at least one value of the interval $\left[a_{j}, a_{k}\right]$ does not appear in $a$. Moreover, we say that $a_{i} a_{j} a_{k}$ is an occurrence of the pattern $\overline{2} 1 \overline{3}$ if it is an occurrence of 213 such that $a_{i}=a_{k}-1 .^{3}$ By interpreting Theorem 7 in terms of partial permutations, we obtain easily:

Theorem 8. A permutation $\pi$ is $(123,312)$-sortable if and only if $\alpha(\pi)$ avoids $31 \mid 2$ and $\overline{2} 1 \overline{3}$.

Let $\mathcal{A}_{n}(31 \mid 2, \overline{2} 1 \overline{3})$ be the set of partial permutations of $n$ avoiding the two patterns $31 \mid 2$ and $\overline{2} 1 \overline{3}$, and $\mathcal{A}_{n}(213)$ be the set of partial permutations of $n$ avoiding the classical pattern 213 .

Theorem 9. For any $n \geq 1$, there is a bijection $\phi$ between $\mathcal{A}_{n}(31 \mid 2, \overline{2} 1 \overline{3})$ and $\mathcal{A}_{n}(213)$.

Proof. Let us define recursively the map $\phi$ from $\mathcal{A}_{n}(31 \mid 2$, $\overline{2} 1 \overline{3})$ to $\mathcal{A}_{n}(213)$. If $\pi=\lambda$, then we set $\phi(\pi)=\lambda$. Otherwise, $\pi$ has a unique decomposition of the form $\pi=$ $A \min (\pi) B$ where $A$ and $B$ are disjoint partial permutations of $n$. We distinguish three cases:

(i) If at least one of $A$ or $B$ is empty, then we set $\phi(\pi)=$ $\phi(A) \min (\pi) \phi(B)$;

(ii) If both $A$ and $B$ are not empty and $\min (A)>\max (B)$, then we set $\phi(\pi)=\phi(A) \min (\pi) \phi(B)$. It is worth noting that the hypothesis that $\pi$ avoids $31 \mid 2$ implies that any value $x \in[\min (\pi), \max (B)]$ occurs in $B$.

(iii) Suppose that both $A$ and $B$ are not empty and $\min (A)<\max (B)$. Since $\pi$ avoids $\overline{2} 1 \overline{3}$, there exists $x \in[\min (A), \max (B)]$ such that $x$ does not occur in $\pi$. We choose the smallest $x$ with this property, so that any value of the interval $[\min (A), x]$ occurs in $A$. Moreover, since $\pi$ avoids $31 \mid 2$, it must be $\max (A)=$ $x-1$. An illustration of this case is depicted in Fig. 1 . Let $r$ be the maximum value of $B$ that is lower than $\min (A)$ and consider the string $B^{\prime}$ obtained from $B$ by decreasing by $x-r-1$ all entries greater than $x$. Similarly, let $A^{\prime}$ be obtained from $A$ by increasing by $\max (B)-x+1$ all its entries. Obviously, $A^{\prime}$ and $B^{\prime}$ belong to $\mathcal{A}_{n}(31 \mid 2, \overline{2} 1 \overline{3})$, whereas $\pi^{\prime}=A^{\prime} \min (\pi) B^{\prime}$ contains $31 \mid 2$. Then we set $\phi(\pi)=\phi\left(A^{\prime}\right) \min (\pi) \phi\left(B^{\prime}\right)$ (see again Fig. 1 for an illustration of this mapping). It is worth noting that the value $r+1$ does not occur in both $A^{\prime}$ and $B^{\prime}$, which implies that there exists $y \in[\min (\pi), r+1]$ such that $y$ does not occur in $\phi\left(B^{\prime}\right)$.

Next we prove that $\phi$ is an injective map. We proceed by induction on the length of partial permutations. Let $\pi$ be a partial permutation. Due to the remarks at the end of (ii) and (iii), the image of $\pi$ under $\phi$ satisfying (ii) is a partial permutation $\pi^{\prime}$ such that any value $x \in[\min (\pi), \max (\phi(B))]$ occurs in $\phi(B)$, which is not true for a permutation $\pi$ satisfying (iii). Then, for two nonempty partial permutations $\pi$ and $\sigma$ in $\mathcal{A}_{n}(31 \mid 2, \overline{2} 1 \overline{3})$,

\footnotetext{
3 This is analogous to the notion of bivincular pattern on classical permutations.
} 


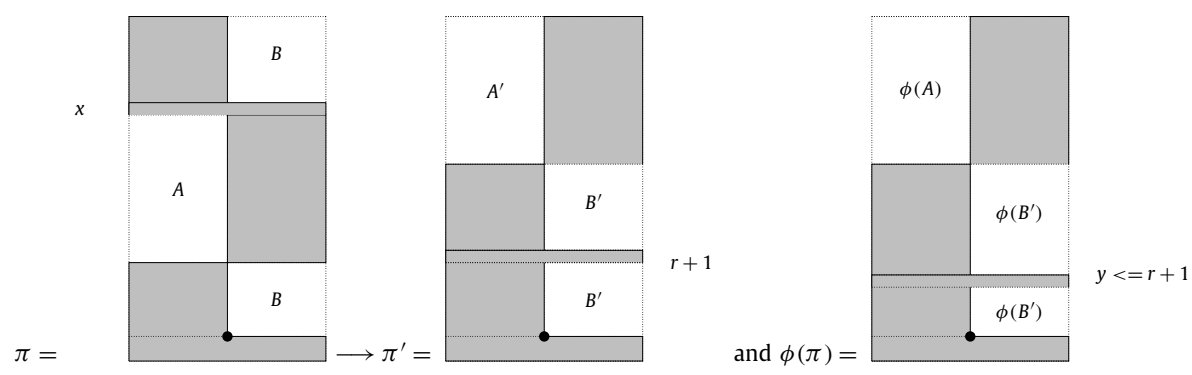

Fig. 1. Illustration of $\phi$ in the case (iii) of the proof of Theorem 8 .

Table 2

Counting sequences not appearing in [15].

\begin{tabular}{c|l}
\hline$(\sigma, \tau)$ & $\begin{array}{l}\text { The sequence counting } \\
(\sigma, \tau) \text {-sortable permutations }\end{array}$ \\
\hline$(123,231)$ & $1,2,6,21,79,310,1252,5168,21714, \ldots$ \\
$(132,213),(213,312)$ & $1,2,5,16,261,1206,5882,29861,156366, \ldots$ \\
$(123,321)$ & $1,2,4,7,14,28,56,112,224,448,896,1792, \ldots$ \\
$(213,231)$ & $1,2,6,23,101,483,2450,12978,71071, \ldots$ \\
$(213,321)$ & $1,2,4,12,46,200,941,4677,24203, \ldots$ \\
$(231,312)$ & $1,2,6,23,101,484,2471,13254,74026, \ldots$ \\
$(312,321)$ & $1,2,4,10,28,85,274,925,3239, \ldots$ \\
\hline
\end{tabular}

$\phi(\pi)=\phi(\sigma)$ implies that $\pi$ and $\sigma$ have the same length and they belong to the same case (i), (ii) or (iii). The recurrence hypothesis induces $\pi=\sigma$ which completes the induction.

Finally, observe that any partial permutation $\pi$ avoiding 213 is of the form $A \min (\pi) B$ where $\min (A)>\max (B)$ and both $A$ and $B$ avoid 213. According to the geometrical shape of $\pi$ (as in the proof of injectivity), $\pi$ fits exactly in one of the cases (i), (ii) and (iii) in the definition of $\phi$. Therefore the surjectivity of $\phi$ can be showed by using its recursive definition and induction on $A$ and $B$. We leave the details to the reader.

Now, it is easy to enumerate the set $\mathcal{A}_{n}$ (213). Indeed any partial permutation $\pi \in \mathcal{A}_{n}(213)$ can be obtained by choosing $k$ integers from $\{1,2, \ldots, n\}$ and then arranging them according to the partial order of a permutation in $\operatorname{Av}_{k}(213)$ (there are $c_{k}$ such permutations). Therefore, we have:

$\left|\mathcal{A}_{n}(213)\right|=\sum_{k=0}^{n}\left(\begin{array}{l}n \\ k\end{array}\right) c_{k}$

the binomial transform of Catalan numbers (sequence A 007317 in [15]). The enumeration of Sort $(123,312)$ follows immediately.

Corollary 4. For each $n \geq 1$, we have:

$\left|\operatorname{Sort}_{n+1}(123,312)\right|=\sum_{k=0}^{n}\left(\begin{array}{l}n \\ k\end{array}\right) c_{k}$.

\section{Final remarks}

We have focused our study on pairs $(\sigma, \tau)$ of patterns of length three for which permutations sortable by $(\sigma, \tau)$-machine are counted by Catalan numbers, by their binomial transform, or by Schröder numbers, thereby addressing six out of fifteen possible pairs of patterns of length three. Computer experiments have shown that for all nine remaining pairs of length three patterns, except for $(132,321)$, the corresponding counting sequences are not recorded in [15] database (see Table 2), and we state the next conjecture.

Conjecture 1. The sequence counting $(132,321)$-sortable permutations is the sequence A1 02407 in [15] counting particular tukasiewicz paths and pattern avoiding Dyck paths.

Moreover, the pairs $(132,213)$ and $(213,312)$ yield the same enumerating sequence.

\section{Declaration of competing interest}

The authors declare that they have no known competing financial interests or personal relationships that could have appeared to influence the work reported in this paper.

\section{Acknowledgements}

The authors are grateful to the anonymous referees for carefully reading the manuscript and providing many useful suggestions. 


\section{References}

[1] J. Bandlow, E.S. Egge, K. Killpatrick, A weight-preserving bijection between Schröder paths and Schröder permutations, Ann. Comb. 6 (3-4) (2002) 235-248.

[2] E. Barcucci, A. Del Lungo, E. Pergola, R. Pinzani, Some combinatorial interpretations of $q$-analogs of Schröder numbers, Ann. Comb. 3 (1999) 171-190.

[3] J.-L. Baril, C. Khalil, V. Vajnovszki, Catalan and Schröder permutations sortable by two restricted stacks, https://arxiv.org/pdf/2004.01812, 2020.

[4] J.-L. Baril, G. Cerbai, C. Khalil, V. Vajnovszki, Permutations sortable by two restricted stacks: Catalan and Schröder cases, in: Permutation Patterns Virtual Workshop, June 30 - July 1, 2020.

[5] K. Berlow, Restricted stacks as functions, https://arxiv.org/pdf/2008. 01164, 2020.

[6] Y. Cai, C. Yan, Counting with Borel's triangle, Discrete Math. 342 (2) (2019) 529-539.

[7] G. Cerbai, A. Claesson, L. Ferrari, Stack sorting with restricted stacks, J. Comb. Theory, Ser. A 173 (2020).
[8] G. Cerbai, A. Claesson, L. Ferrari, E. Steingrímsson, Sorting with pattern-avoiding stacks: the 132-machine, Electron. J. Comb. 27 (3) (2020).

[9] G. Cerbai, Sorting Cayley permutations with pattern-avoiding machines, https://arxiv.org/pdf/2003.02536, 2020.

[10] C. Defant, K. Zheng, Stack-sorting with consecutive-pattern-avoiding stacks, https://arxiv.org/pdf/2008.12297, 2020.

[11] D. Desantis, R. Field, W. Hough, B. Jones, R. Meissen, J. Ziefle, Permutation pattern avoidance and the Catalan triangle, Mo. J. Math. Sci. 25 (1) (2013) 50-60.

[12] S. Kitaev, Patterns in Permutations and Words, Springer, 2011.

[13] D.E. Knuth, The Art of Computer Programming, Vol. 1, Fundamental Algorithms, Addison-Wesley, 1968.

[14] L.W. Shapiro, A Catalan triangle, Discrete Math. 14 (1976) 83-90.

[15] N.J.A. Sloane, The on-line encyclopedia of integer sequences, http:// oeis.org/.

[16] J. West, Generating trees and the Catalan and Schröder numbers, Discrete Math. 146 (1995) 247-262. 